\title{
VARIÁVEIS PSICOLÓGICAS ASSOCIADAS À ADESÃO AO TRATAMENTO DA DIABETES MELLITUS ${ }^{1}$
}

\author{
Isabel Silva ${ }^{2}$ \\ José Pais-Ribeiro ${ }^{3}$ \\ Helena Cardoso ${ }^{4}$
}

Resumo: O presente estudo, de carácter exploratório e transversal, teve como objectivo identificar e analisar variáveis psicológicas associadas à adesão aos autocuidados da diabetes. Foi avaliada uma amostra de conveniência de 316 indivíduos com diabetes, dos quais $44,6 \%$ do sexo masculino; com idades compreendidas entre os 16 e os 84 anos $(M=48,39 ; D P=16,90)$; $41,8 \%$ apresenta diagnóstico de diabetes tipo 1 e a duração da doença varia entre 4 meses e 43 anos $(M=13,66 ; D P=9,32)$. A análise dos dados sugere que a satisfação com o apoio social está modesta e positivamente correlacionada com a adesão aos autocuidados. As estratégias de coping instrumentais revelam estar modesta e positivamente correlacionadas com a adesão, ao contrário da ansiedade, que está modesta e negativamente correlacionada com os autocuidados da diabetes. Os dados sugerem, ainda, que o stresse e depressão não estão correlacionados de forma estatisticamente significativa com a gestão desta doença.

Palavras-chave: Adesão ao tratamento, diabetes, variáveis psicológicas.

Psychological variables associated to treatment adherence in diabetes mellitus (Abstract): The objective of the present exploratory and transversal study is to identify and analyse psychological variables that can be related to treatment adherence in diabetic patients. A convenience sample included 316 diabetic patients; 44,6\% males; aged between 16 and 84 years $(M=48,39 ; S D=16,90)$, $41,8 \%(n=132)$ with type 1 diabetes and 58,2\% $(n=184)$ with type 2 diabetes. Disease duration varies between 4 months e 43 years $(M=13,66 ; S D=9,32)$ and $59,8 \%$ of those patients suffers from diabetes chronic complications. Results suggest that satisfaction with social support and instrumental coping strategies are

\footnotetext{
${ }^{1}$ Trabalho realizado com o apoio da bolsa Praxis XXI BD/21804/9 da Fundação para a Ciência e a Tecnologia.

2 Rua da Constituição 2174, 2. ${ }^{\circ}$ esq, 4200 Porto; e-mail: ilopessilva@hotmail.com

${ }^{3}$ Faculdade de Psicologia e de Ciências da Educação da Universidade do Porto, Rua do Campo Alegre, 1055, 4169-004 Porto.

${ }^{4}$ Hospital Geral de Santo António - Serviço de Endocrinologia - Largo Prof. Abel Salazar, 4099-001 Porto.
} 
modestly and positively correlated to self-care activities. Positive and negative life events, as well as total stress and depression, are not significantly correlated with treatment adherence in diabetic patients. Finally, anxiety symptoms showed to be modestly and negatively related to diabetes self-care activities adherence.

\section{Introdução}

O tratamento da diabetes implica um complexo conjunto de acções comportamentais de cuidados numa base diária, ao longo de toda a vida do doente, e é amplamente reconhecido como sendo extremamente desafiante pelo grau de envolvimento que exige ao doente.

À semelhança do que se verifica com outras doenças crónicas, também a não adesão ao tratamento da diabetes tem demonstrado ser elevada. Este facto tem estimulado o desenvolvimento de numerosos estudos, que se têm debruçado sobre a identificação e análise de determinantes da adesão ao tratamento nesta população.

\section{A natureza multifactorial da adesão ao tratamento}

A investigação tem revelado que a adesão tem uma natureza multifactorial, sendo influenciada por variáveis que actuam a partir de fontes diversas (Leventhal \& Cameron, 1987).

Em 1976, Sackett e Haynes identificaram mais de duzentas variáveis relacionadas com a adesão ao tratamento, que agruparam em cinco categorias: (1) características do doente; (2) características do tratamento; (3) características da doença; (4) relacionamento com os profissionais de saúde; (5) e variáveis organizacionais e estruturais.

Mais recentemente, Sanchez-Sosa (2001) propôs uma classificação em quatro componentes: (1) o doente; (2) a equipa de saúde; (3) a doença em si; (4) e factores associados às condições do macro-sistema, relacionados com traços socioeconómicos, geográficos e culturais de um determinado país.

A literatura sugere ser muito pouco provável que algum destes grupos de factores actue de forma isolada e que factores pertencentes a diferentes fontes devem interagir, produzindo vários graus de adesão ao tratamento. 
O presente estudo propõe debruçar-se essencialmente sobre algumas variáveis psicológicas que têm demonstrado desempenhar um papel relevante na adesão ao tratamento da diabetes.

\section{O humor negativo}

Uma variável que tem sido alvo de amplo estudo no domínio da adesão ao tratamento é o humor negativo. A literatura aponta para que, nas crianças e nos adultos, a presença de perturbações psicológicas, como depressão e ansiedade, esteja associada a uma má gestão da diabetes, apesar de nem todos os estudos replicarem essa relação (Nagasawa, Smith, Barnes \& Fincham, 1990). Sherbourne, Hays, Ordway, DiMatteo e Kravitz (1992) verificaram que os doentes que se sentiam mais perturbados com a sua saúde ou que apresentavam pior funcionamento físico e pior desempenho físico (extensão com que a saúde física interfere no tipo e quantidade de trabalho ou actividades do quotidiano) tinham, de uma forma geral, menor probabilidade de aderir aos autocuidados da diabetes. Também Ryan (1997) considera que os estados emocionais podem influenciar o controlo metabólico directamente, interferindo via sistema nervoso autonómico, ou indirectamente, interferindo com a capacidade da pessoa gerir a sua doença.

\section{Competências de coping}

As competências de coping dos doentes, quer em termos de resolução de problemas específicos da diabetes, quer de capacidade para lidar com o stress da vida, podem influenciar os autocuidados e o resultado do tratamento (Cox \& Gonder-Frederick, 1992; Ryan, 1997). Por exemplo, Sherbourne e colaboradores (1992) constataram que os doentes mais jovens e que usam estratégias de coping evitantes tendem a seguir menos as recomendações específicas dos médicos.

\section{Apoio social}

O papel do apoio social como preditor da adesão aos autocuidados da diabetes tem, igualmente, sido alvo de interesse dos investigadores. A complexidade das relações entre as interacções familiares e a adesão ao tratamento ainda está muito pouco estudada nos adultos, sendo que a maioria dos estudos realizados até à data se tem debruçado sobre a criança diabética e a sua família. Os resultados da investigação neste domínio são 
contraditórios. Alguns autores não encontram qualquer relação significativa entre o apoio social e a adesão ao tratamento em indivíduos com diabetes tipo 2 (Chlebowy, 2002). Todavia, outros estudos sugerem que a relação conjugal parece funcionar, quer como uma importante fonte de apoio social, quer como uma potencial origem de stress e que a inclusão de outros significativos em programas de cuidados de saúde tem demonstrado melhorar significativamente a adesão ao tratamento da diabetes. Quando a comunicação familiar é praticamente inexistente, é provável que o apoio (como, por exemplo, lembrar que são horas da medicação, que deve comer uma menor quantidade ou comer em determinado horário) não esteja de acordo com o que o doente espera, sendo percebido como crítica, gerando ressentimento e conduzindo directa e indirectamente à não adesão, depressão, ansiedade e stress (Warren \& Hixenbaugh, 1998).

Apesar do ambiente social ter demonstrado ser importante para o controlo da diabetes, o papel dos outros significativos fora da família continua pouco claro. Quando usada uma medida geral de apoio social, os homens com bom apoio social dos amigos, ao contrário das mulheres, apresentam mau controlo metabólico. Porém, quando é utilizada uma escala de apoio social específica para a diabetes, a percepção de que se dispõe de bom apoio social parece estar relacionada com a não adesão, quer nos homens, quer nas mulheres. A função de apoio social dos colegas de trabalho nos homens e mulheres com diabetes é desconhecida, bem como o impacto da diabetes nas suas carreiras (Warren \& Hixenbaugh, 1998).

Cox e Gonder-Frederick (1992) consideram que não é surpreendente que as relações e interacções familiares possam aumentar ou diminuir a adesão ao tratamento da diabetes. Alguns estudos têm sugerido que o conflito e disfunção familiar predizem uma pior adesão ao tratamento, enquanto níveis elevados de apoio social, coesão e organização estão associados a uma melhor adesão aos autocuidados e controlo metabólico (Cox \& Gonder-Frederick, 1992; Sherbourne et al., 1992).

Garay-Sevilla e colaboradores (1995) constataram que o apoio social é o preditor mais forte e consistente dos diferentes comportamentos de autocuidados, nomeadamente da adesão à medicação, nos indivíduos com diabetes tipo 2. Nestes doentes, o apoio social está também associado a uma maior adesão à dieta, autocuidado que beneficia de um maior apoio dos familiares, uma vez que, frequentemente, família e doente partilham as refeições, sobretudo nas sociedades mais tradicionais.

Estes investigadores verificaram que os doentes com famílias que exercem um controlo mais rígido apresentam uma menor adesão à medicação e uma diminuição da adaptação à mudança, em particular às mudanças negativas. $\mathrm{O}$ controlo rígido promove, também, o desenvolvimento de conflitos com a autoridade, aumentando a negação da doença, aspectos que se 
reflectem na não adesão ao tratamento. Por outro lado, o desenvolvimento de uma doença com forte carga genética tem um grande impacto na família, podendo alterar a capacidade de adaptação desta e levando-a a adoptar formas de controlo mais rígidas, em resposta à ameaça da sua desintegração. Este estudo sugere que um ambiente de controlo mais flexível está associado a uma maior adesão ao tratamento e à percepção de um maior apoio social (Garay-Sevilla et al., 1995).

Garay-Sevilla e colaboradores (1995) verificaram, ainda, que ter cônjuges com idades mais avançadas está associado a uma melhor adesão à medicação, o que pensam poder indicar que o tempo pode melhorar os factores associados à aceitação da doença e do tratamento e às condições das famílias destes indivíduos.

\section{Outros determinantes psicológicos da adesão ao tratamento}

Os traços de personalidade também têm sido associados à adesão ao tratamento da diabetes. A investigação tem sugerido que as características da personalidade predizem o controlo metabólico em adultos (Basco, 1998; Lustman, Frank \& McGill, 1991; Ryan, 1997).

Também o locus de controlo tem sido analisado enquanto variável susceptível de influenciar a adesão. Ryan (1997) verificou que os doentes com um locus de controlo interno, isto é, que crêem que são responsáveis pela sua saúde, tendem a apresentar uma melhor adesão ao tratamento e um melhor controlo metabólico do que os doentes com um locus de controlo externo, que crêem estar à mercê do destino ou de qualquer outra força exterior.

Se, nos anos 80, Schlenk e Hart (1984) consideravam que a relação entre crenças de saúde e adesão ao tratamento da diabetes ainda não tinha sido suficientemente demonstrada de forma a fornecer preditores clínicos úteis, nos anos 90, as dúvidas parecem ter-se dissipado. As crenças de saúde, a percepção de susceptibilidade, controlo, auto-eficácia e a expectativa de resultados (benefícios/barreiras) têm demonstrado estar correlacionados com a adopção e manutenção de comportamentos específicos de adesão aos autocuidados em adultos com diabetes e os procedimentos utilizados para a mudança destas variáveis têm demonstrado ser úteis para aumentar a adesão ao tratamento (Brooks, 2002; Cox \& Gonder-Frederick, 1992; Cox et al., 1991; Hampson, 1998; Johnston-Brooks, Lewis \& Garg, 2002; Lutfey \& Wishner, 1999; Skinner, Hampson \& Fife-Schaw, 2002; Wagner, Schnoll \& Gipson, 1998; Watts, 1980; Zgibor \& Simmons, 2002). 
$\mathrm{O}$ autoconceito e a auto-estima também foram relacionados com a adesão, nomeadamente com a adesão à automonitorização da glicemia (Wagner et al., 1998).

Alguns estudos têm confirmado a importância da auto-eficácia na adesão ao tratamento na diabetes. Senécal, Nouwen e White (2000) referem que a revisão da literatura demonstra que a elevada auto-eficácia está associada a uma maior satisfação com o tratamento e com um melhor controlo metabólico e que as intervenções que aumentam a auto-eficácia ao nível da alimentação resultam num aumento da adesão aos cuidados alimentares e numa maior capacidade para superar os obstáculos à implementação dos autocuidados que vão surgindo. Todavia, outros estudos não encontram qualquer relação significativa entre a auto-eficácia e a adesão a comportamentos de autocuidados em indivíduos com diabetes tipo 2 (Chlebowy, 2002).

Um outro aspecto do doente com diabetes que tem sido alvo de estudo, sobretudo na última década, é a presença de perturbações do comportamento alimentar, que, nesta população, tem consequências ainda mais graves do que na população em geral (Crow, Keel, \& Kendall, 1998; Rodin, Craven, Littlefield, Murray \& Daneman, 1991; Rodin et all., 2002; Schwartz, Weissberg-Benchell \& Perlmuter, 2002).

Também a fobia ou medo a injecções ou picadas se tem revelado um fenómeno que poderá prejudicar o tratamento insulínico e a automonitorização da glicemia capilar, aumentar a perturbação associada à diabetes, diminuir a qualidade de vida e aumentar a co-morbilidade psicológica (Mollema, Snoek, Heine, \& van der Ploeg, 2001).

O presente estudo teve como objectivo identificar e analisar variáveis psicológicas associadas à adesão aos autocuidados da diabetes.

\section{Método}

\section{Participantes}

Os participantes constituem uma amostra de conveniência de 316 sujeitos com diabetes, dos quais $44,6 \%$ do sexo masculino; com idades compreendidas entre os 16 e os 84 anos $(M=48,39 ; D P=16,90) ; 17,7 \%$ solteiros; $72,5 \%$ casados/união de facto; $3,8 \%$ divorciados/separados; $6 \%$ viúvos. Dos participantes, $41,8 \%(n=132)$ apresenta diagnóstico de diabetes tipo 1 e $58,2 \%(n=184)$ de diabetes tipo 2 , e a duração da doença varia entre 4 meses e 43 anos $(M=13,66 ; D P=9,32)$. Destes doentes, 59,8\% sofre de complicações crónicas da diabetes. 


\section{Material}

\section{Questionário de Autocuidados da Diabetes}

Instrumento de auto-resposta, com tipo de resposta ordinal de 5 ou 6 posições, que visa avaliar a adesão ao tratamento na diabetes ao longo da última semana. É constituído por 11 itens, que se agrupam em três dimensões: cuidados alimentares, tratamento insulínico e tratamento com antidiabéticos orais. Apresenta uma fidelidade aceitável. e uma validade convergente/discriminante dos itens razoável (Silva, Pais-Ribeiro, Cardoso, \& Ramos, 2002).

\section{Escala de Satisfação com o Suporte Social (ESSS)}

Escala de auto-resposta desenvolvida por Ribeiro (1999) e que avalia a satisfação que o indivíduo sente em relação ao apoio social que pensa ter disponível, nomeadamente em relação a quatro domínios: satisfação com a família, com as amizades, com a intimidade e com as relações sociais. A escala revela ser facilmente compreendida pelos respondentes, de resposta rápida e bem aceite. Trata-se de uma escala fiel e com boa validade convergente-discriminante.

Escala de Coping com Problemas de Saude (Coping with Health Problems and Injuries - CHIP)

Escala de auto-resposta desenvolvida por Endler, Parker e Summerfeldt (1998). Trata-se de uma escala multidimensional de coping para indivíduos que experimentam diferentes problemas de saúde. Optou-se por eliminar a subescala de coping paliativo, na versão utilizada no presente estudo, uma vez que apresentava uma baixa consistência interna (possivelmente porque os itens construídos com a intenção de avaliar estas estratégias para lidar com problemas de saúde se confundem facilmente com consequências associadas ao mau controlo glicémico, nomeadamente com sintomas relacionados com crises hipoglicémicas e hiperglicémicas) e alguns itens das restantes subescalas. Considerou-se, assim, os seguintes 6 itens como integrantes da subescala coping instrumental: item 3, item 7 , item 11, item 15, item 23 e item 31. Na subescala coping emocional, conservaram-se apenas os seguintes 5 itens: item 4, item 8, item 12, item 16 e item 24. Em relação à subescala de coping distracção, conservaram-se os 8 itens da versão portuguesa da escala: item 1, item 5, item 9, item 13, item 17, item 21, item 25 e item 29. A escala com esta nova estrutura demonstrou apresentar propriedades psicométricas aceitáveis, tratando-se de um instrumento fiel e com uma aceitável consistência interna. 
Avaliação de Acontecimentos de Vida (Life Experiences Survey - LES)

Foi utilizada a versão portuguesa do LES (Silva, Pais-Ribeiro, Cardoso \& Ramos, para publicação). A escala foi originalmente desenvolvida por Sarason, Johnson e Siegel, em 1978, e trata-se de um instrumento de auto-relato constituído por 47 itens, mais 3 espaços em branco nos quais o respondente pode indicar outros acontecimentos que possa ter experimentado ao longo do último ano. Este instrumento tem como objectivo avaliar a frequência e o tipo (positivo quando o acontecimento é desejável ou negativo quando indesejável) e intensidade do impacto dos acontecimentos de vida ocorridos no último ano, apresentando uma boa fidelidade.

Escala de Ansiedade e Depressão Clínica (Hospital Anxiety and Depression Scale - HADS)

Escala de auto-resposta desenvolvida por Zigmond e Snaith, em 1983, e que tem como objectivo identificar, de forma válida, fiel e prática, sintomas de depressão e ansiedade. Apresenta como importante vantagem sobre outras escalas o facto de ter sido concebida de forma a evitar indicadores físicos, que pudessem induzir em erro na avaliação de populações médicas. A versão portuguesa da escala (ainda em estudo - J. Ribeiro, M. Baltar, T. Ferreira, R. Meneses, T. Martins, C. Samarina \& I. Silva.) revela ser de fácil compreensão, resposta rápida e bem aceite pelos respondentes. Trata-se de uma escala fiel e possui uma razoável validade convergente-discriminante.

\section{Procedimento}

As informações clínicas e demográficas foram recolhidas a partir dos processos médicos dos doentes e estes responderam aos questionários no contexto de uma entrevista pessoal, após obtenção do seu consentimento informado. $\mathrm{O}$ consentimento informado, assim como a autorização para a realização do estudo, foram obtidos de acordo com a Declaração de Helsínquia, pela qual o hospital onde os dados foram recolhidos se rege. Esta declaração reconhece o direito à autodeterminação de cada indivíduo e visa garantir o cumprimento das normas éticas da investigação clínica, a responsabilidade científica e profissional, o respeito pelos direitos humanos e pela dignidade da pessoa, a salvaguarda dos interesses do doente e a reputação da instituição em que o estudo foi realizado.

\section{Resultados}

A análise descritiva dos resultados é apresentada no quadro 1.

A análise dos dados sugere que a satisfação com as amizades $[r(302)=0,14 ; p<0,05]$ e com a família $[r(302)=0,14 ; p<0,05]$ está mo- 
desta e positivamente correlacionada com a adesão aos autocuidados alimentares. Todavia, a adesão aos antidiabéticos orais e à insulinoterapia não está significativamente correlacionada, do ponto de vista estatístico, com a satisfação com o apoio social.

O stresse positivo, negativo e globalmente considerado não demonstrou estar significativamente correlacionado, do ponto de vista estatístico, com a adesão a qualquer uma das actividades de autocuidados. Porém, as estratégias adoptadas para lidar com a doença, stresse específico, demonstram estar significativamente correlacionadas com a adesão. A adopção de estratégias de coping instrumentais revelou estar modesta e positivamente correlacionada com a adesão à dieta $[r(304)=0,28 ; p<0,0001]$ e à insulinoterapia $[r(210)=0,15 ; p<0,05]$, ainda que as restantes estratégias de coping não provassem estar significativamente correlacionadas com os autocuidados.

Quanto ao humor negativo, os resultados demonstram que a presença de sintomas de depressão e o humor negativo globalmente considerado não estão significativamente correlacionados com a adesão ao tratamento da diabetes, mas revelam, também, que a presença de sintomas de ansiedade está modesta e negativamente correlacionada com os auto-cuidados desta doença crónica $[r(305)=-17 ; p<0,01]$.

Quadro 1 - Análise descritiva dos resultados

\begin{tabular}{lccccc}
\hline & $\boldsymbol{N}$ & $\begin{array}{l}\text { Limite } \\
\text { mínimo }\end{array}$ & $\begin{array}{l}\text { Limite } \\
\text { máximo }\end{array}$ & $\boldsymbol{M}$ & $\boldsymbol{D P}$ \\
\hline Adesão à Alimentação & 307 & 6,00 & 32,00 & 22,96 & 5,36 \\
Adesão à Insulina & 213 & 0,00 & 8,00 & 7,37 & 1,37 \\
$\begin{array}{l}\text { Adesão aos Antidiabéticos } \\
\quad \text { Orais }\end{array}$ & 139 & 0,00 & 8,00 & 7,40 & 1,52 \\
$\begin{array}{l}\text { Satisfação com a Amizade } \\
\text { Satisfação com a Intimidade }\end{array}$ & 313 & $-10,00$ & 10,00 & 5,44 & 5,15 \\
Satisfação com a Família & 312 & $-8,00$ & 8,00 & 3,69 & 4,17 \\
Satisfação com as & 313 & $-6,00$ & 6,00 & 3,68 & 3,28 \\
$\quad$ Actividades Sociais & & $-6,00$ & 6,00 & 0,79 & 3,92 \\
Total do Apoio Social & 316 & $-30,00$ & 30,00 & 13,48 & 12,55 \\
Coping Emocional & 304 & 1,00 & 5,00 & 2,69 & 0,91 \\
Coping por Distracção & 309 & 1,00 & 5,00 & 3,19 & 0,71 \\
Coping Instrumental & 315 & 1,00 & 5,00 & 3,56 & 0,84 \\
Stresse Positivo & 316 & 0,00 & 37,00 & 5,00 & 5,77 \\
Stresse Negativo & 316 & $-4,00$ & 37,00 & 5,47 & 6,29 \\
Stresse Total & 316 & 0,00 & 43,00 & 6,02 & 4,49 \\
Ansiedade & 316 & 0,00 & 21,00 & 8,16 & 4,57 \\
Depressão & 316 & 0,00 & 21,00 & 5,44 & 4,39 \\
Total do Humor Negativo & 316 & 0,00 & 38,00 & 13,60 & 7,59 \\
\hline
\end{tabular}




\section{Discussão}

A análise dos resultados sugere que a adesão ao tratamento no grupo estudado é, de uma forma geral, elevada. Por outro lado, a Satisfação com os diferentes domínios do apoio social revela ser mais elevada em relação à Família, seguindo-se a satisfação com a Amizade e com a Intimidade, sendo os valores mais baixos encontrados no domínio satisfação com as Actividades Sociais. Os doentes parecem adoptar mais estratégias de coping instrumentais e por distracção do que estratégias de coping emocional. Os resultados sugerem, ainda, que o stresse positivo, negativo e total, ao longo do último ano, é, de forma geral, baixo. Finalmente, no que diz respeito ao nível dos sintomas de ansiedade, este revela ser mais elevado do que o nível dos sintomas de depressão. O nível de ansiedade poderá ser classificado como sendo fraco, enquanto os sintomas de depressão são considerados como encontrando-se dentro de níveis normais, de acordo com os valores de referência apresentados por Snaith e Protheroe (1995), o que sugere que a amostra em estudo não revela apresentar níveis de humor negativo elevados.

O presente estudo sugere que o apoio social, as estratégias de coping instrumental adoptadas para lidar com os problemas de saúde e a ansiedade estão modestamente correlacionados com a adesão aos autocuidados da diabetes, o que poderá sublinhar a natureza multifactorial da adesão ao tratamento da diabetes.

Os dados sugerem, ainda, que, ao contrário do que seria natural esperar, o stresse e depressão não estão correlacionados de forma estatisticamente significativa com a gestão desta doença. Tal poderá levar-nos a colocar a hipótese de que, quanto maior a duração da diabetes, maior poderá ser a possibilidade dos auto-cuidados desta doença serem integrados no estilo de vida do indivíduo e, por isso, a adesão ao tratamento não sofrer um impacto directo de perturbações psicológicas esporádicas. Por outro lado, devemos sublinhar que, no presente estudo, o stresse foi avaliado de uma forma geral, não contemplando factores específicos para a diabetes e gestão desta doença, e que o nível de depressão se encontra, globalmente, dentro de níveis considerados normais.

É muito provável que o apoio social, as estratégias para lidar com problemas de saúde e humor negativo afectem a adesão aos autocuidados da diabetes, de formas distintas, conforme o momento da evolução da doença. Assim, a importância que cada uma destas variáveis psicológicas poderá ter para a adesão será diferente conforme se trate do momento que imediatamente sucede ao diagnóstico de diabetes mellitus, do período posterior de adaptação a esta doença, do confronto com o desenvolvimento de complicações crónicas e do seu tratamento ou do período em que as 
sequelas atingem um nível de maior gravidade, originando grave incapacidade física. $\mathrm{O}$ desenvolvimento de estudos de carácter longitudinal torna-se, pois, premente e imprescindível.

Um outro aspecto que poderá enriquecer profundamente a compreensão sobre a relação entre estas variáveis psicológicas e a adesão ao tratamento da diabetes é a combinação de instrumentos que incluam quer itens gerais, quer itens específicos da diabetes para avaliar estes construtos. Uma das limitações do presente estudo prende-se precisamente com a utilização exclusiva de instrumentos de avaliação genéricos (ex., apoio social, stresse, coping, ansiedade e depressão), com excepção do instrumento de adesão ao tratamento. Será essencial analisar a forma como o apoio social específico para a diabetes e seu tratamento, o stress a estes associado e as estratégias de coping específicas para lidar com a diabetes mellitus se relacionam ao longo do tempo com a adesão aos autocuidados.

Os resultados do presente estudo sugerem que será importante, na prática clínica, não só psicólogos, como outros profissionais de saúde ligados ao diagnóstico, tratamento e educação na área da diabetes, valorizarem aspectos relacionados com a satisfação que estes doentes revelam face ao apoio social, com as estratégias de coping adoptadas para lidar com os problemas de saúde e com a ansiedade, uma vez que constituem factores relevantes na adesão ao tratamento da diabetes e, consequentemente, na prevenção de complicações crónicas e agudas desta doença.

\section{Referências}

Basco, M. R. (1998). Perfectionism and diabetes care. Diabetes Spectrum, 11 (1), 43-48.

Brooks, L. M. (2002). Perceived barriers to treatment adherence among pregnant African American women with diabetes. Dissertation submitted in partial fulfillment of the requirements for the degree of Doctor of Philosophy. U.S.A.: Case Western Reserve University (Health Sciences).

Chlebowy, D. O. (2002). Impact of social support, self-efficacy, and outcome expectations on self-care behaviors and glycemic control in Caucasian and African-American adults with type 2 diabetes mellitus. Dissertation presented in the fulfillment of the requirements for the degree of Doctor of Philosophy. U.S.A.: Graduate School of the Ohio State University.

Cox, D. J. \& Gonder-Frederick (1992). Major developmentes in behavioral diabetes research. Journal of Consulting and Clinical Psychology, 60 (4), 628-638.

Cox, D. J., Gonder-Frederick, L., \& Saunders, J. T. (1991). Diabetes: clinical issues and management. In J. Sweet, R. Rosensky, \& S. Tovian (Eds.), Handbook of clinical psychology in medical settings (pp. 473-496). Plenum Press. 
Crow, S. J., Keel, P. K. \& Kendall, D. (1998). Eating disorders and insulin-dependent diabetes mellitus. Psychosomatics, 39, 233-243.

Endler, N. S., Parker, J. D. A., \& Summerfeldt, L. J. (1998). Coping with health problems: developing a reliable and valid multidimensional measure. Psychological Assessment, 10 (3), 195-205.

Garay-Sevilla, M. E., Nava, L. E., Malacara, J. M., Huerta, R., de Léon, J. D., Mena, A., \& Fajardo, M.E. (1995). Adherence to treatment and social support in patients with non-insulin dependent diabetes mellitus. Journal of Diabetes and Its Complications, 9, 81-86.

Hampson, S. E. (1998). Illness representations and the self-management of diabetes. In K. J. Petrie, \& J. A. Weinman (Eds.), Perceptions of health \& illness (pp. 323-347). The Netherlands: Harwood Academic Publishers.

Johnston-Brooks, C. H., Lewis, M A., \& Garg, S. (2002). Self-efficacy impacts self-care and HbA1c in Young adults with type I diabetes. Psychosomatic Medicine, 64, 43-51.

Leventhal, H., \& Cameron, L. (1987). Behavioral theories and the problem of compliance. Patient Education and Counseling, 10, 117-138.

Lustman, P. J., Frank, B. L., \& McGill, J. B. (1991). Relationship of personality characteristics to glucose regulation in adults with diabetes. Psychosomatic Medicine, 53 (3), 305-312.

Lutfey, K. E., \& Wishner, W. J. (1999). Beyond "compliance" is "adherence". Diabetes Care, 22 (4), 635-639.

Mollema, E. D., Snoek, F.J., Heine, R. J., \& van der Ploeg, H. M. (2001). Insulin-treated diabetes patients with fear of self-injecting or fear of self-testing: Psychological comorbidity and general well-being. Journal of Psychosomatic Research, 51 (5), 665-672.

Nagasawa, M., Smith, M. C., Barnes, J. H., \& Fincham, J. E. (1990). Meta-analysis of correlates of diabetes patients' compliance with prescribed medications. The Diabetes Educator, 16, 192-200.

Ribeiro, J. L. P. (1999). Escala de satisfação com o suporte social. Análise Psicológica, 3 (8), 547-558.

Rodin, G., Craven, J., Littlefield, C., Murray, M., \& Daneman, D. (1991). Eating disorders andintentional insulin undertreatment in adolescent females with diabetes. Psychosomatics, 32, 171-176.

Rodin, G., Olmsted, M. P., Rydall, A. C., Maharaj, S.I., Colton, P. A., Jones, J. M., Biancucci, L. A., \& Daneman, D. (2002). Eating disorders in young women with type 1 diabetes mellitus. Journal of Psychosomatic Research, 53, 943-949 .

Ryan, C. M. (1997). Psychological factors and diabetes mellitus. In J. Pickup \& G. Williams (Eds.), Textbook of diabetes (pp. 66.1-66.13). Oxford: Blackwell Science.

Sackett, D. L., \& Haynes, R. B. (Eds.) (1976). Compliance with therapeutic regimens. Baltimore, MD.: Johns Hopkins University Press.

Sanchez-Sosa, J. J. (2001). Treatment adherence: the role of behavioral mechanisms operating through health care interventions. Comunicação apresentada no 
Meeting Adherence to Long-Term Therapies: Toward Policies for Action, World Health Organization, Geneva.

Sarason, I. G., Johnson, J. H., \& Siegel, J. M. (1978). Assessing the impact of life changes: Development of the life experiences survey. Journal of Consulting and Clinical Psychology, 46 (5), 932-946.

Schlenk, E. A., \& Hart, L. K. (1984). Relationship between health locus of control, health value and social support and compliance with diabetes mellitus. Diabetes Care, 7, 566-574.

Schwartz, S. A., Weissberg-Benchell, J., \& Perlmuter, L. C. (2002). Personal control and disordered eating in female adolescents with type 1 diabetes. Diabetes Care, 25, 1987-1991.

Senécal, C., Nouwen, A. \& White, D. (2000). Motivation and dietary self-care in adults with diabetes: Are self-efficacy and autonomous self-regulation complementary or competing constructs? Health Psychology, 19 (5), 452-457.

Sherbourne, C. D., Hays, R. D., Ordway, L., DiMatteo, M. R., \& Kravitz, R. L. (1992). Antecedents of adherence to medical recommendations: Results from the Medical Outcomes Study. Journal of Behavioral Medicine, 15 (5), 447-468 .

Silva, I., Pais-Ribeiro, J., Cardoso, H., \& Ramos, H. (2002). Questionário de auto-cuidados na diabetes - Contributo para a criação de um instrumento de avaliação da adesão ao tratamento. Psiquiatria Clínica, 23 (3), 227-237.

Silva, I., Pais-Ribeiro, J., Cardoso, H., \& Ramos, H. (para publicação). Contributo para a adaptação da Life Experiences Survey (LES) à população diabética portuguesa. Revista Portuguesa de Saúde Pública.

Skinner, T. C., Hampson, S. E., \& Fife-Schaw, C. (2002). Personality, personal model beliefs, and self-care in adolescents and young adults with type 1 diabetes. Health Psychology, 21, 1, 61-70.

Snaith, R. P., \& Protheroe, D. N. (1995). The hospital anxiety and depression scale. Hospital Update, May, 240-242.

Wagner, J. A., Schnoll, R. A., \& Gipson, M. T. (1998). Development of a scale to measure adherence to self-monitoring of blood glucose with latent variable measurement. Diabetes Care, 21 (7), 1046-1051.

Warren, L., \& Hixenbaugh, P. (1998). Adherence and diabetes. In LB. Myers \& K. Midence (Eds.), Adherence to treatment in medical conditions (pp. 423-453). Netherlands: Harwood Academic Publishers.

Watts, F. N. (1980). Behavioural aspects of the management of diabetes mellitus: education, self-care and metabolic control. Behavior Research \& Therapy, 18, 171-180.

Zgibor, J. C., \& Simmons, D. (2002). Barriers to blood glucose monitoring in a multiethnic community. Diabetes Care, 25 (10), 1772-1777.

Zigmond, A. S., \& Snaith, R P. (1983). The hospital anxiety and depression scale. Acta Psychiatrica Scandinavica, 67, 361-370. 